Draft VERSION NOVEMBER 5, 2018

Preprint typeset using $\mathrm{L}^{A} \mathrm{~T} \mathrm{E}$ X style emulateapj v. 11/10/09

\title{
SUBMILLIMETER GALAXY NUMBER COUNTS AND MAGNIFICATION BY GALAXY CLUSTERS
}

\author{
Marcos Lima, Bhuvnesh Jain, Mark Devlin, and James Aguirre \\ Department of Physics \& Astronomy, University of Pennsylvania, Philadelphia PA 19104 \\ Draft version November 5, 2018
}

\begin{abstract}
We present an analytical model which reproduces measured galaxy number counts from surveys in the wavelength range of $500 \mu \mathrm{m}$ to $2 \mathrm{~mm}$. The model involves a single high-redshift galaxy population with a Schechter luminosity function which has been gravitationally lensed by galaxy clusters in the mass range $10^{13}$ to $10^{15} M_{\odot}$. This simple model reproduces both the low flux and the high flux end of the number counts reported by the BLAST, SCUBA, AzTEC and the SPT surveys. In particular, our model accounts for the most luminous galaxies detected by SPT as the result of high magnifications by galaxy clusters (magnification factors of 10-30). This interpretation implies that submillimeter and millimeter surveys of this population may prove to be a useful addition to ongoing cluster detection surveys. The model also implies that the bulk of submillimeter galaxies detected at wavelengths larger than $500 \mu \mathrm{m}$ lie at redshifts greater than 2 .

Subject headings: galaxies: clusters — gravitational lensing — submillimeter: galaxies
\end{abstract}

\section{INTRODUCTION}

Over the last decade, submillimeter (submm) surveys have yielded significant advances in our understanding of the galaxy population responsible for the high-redshift component of the cosmic infrared background (CIB) (Fixsen et al. 1996; Smail et al. 1997: Hughes et al. 1998; Barger et al. 1998; Dwek et al. 1998; Fixsen et al. 1998; Greve et al. 2004; Pope et al. 2006; Coppin et al. 2006; Devlin et al. 2009). With typical far-infrared (FIR) luminosities $>10^{12} L_{\odot}$, submm galaxies are presumed to be the high-redshift counterparts to (ultra) luminous infrared galaxies (LIRGs, ULIRGs). The high luminosity of these galaxies is the result of star formation rates of $100-1000 M_{\odot} \mathrm{yr}^{-1}$. Approximately half of these galaxies are located at $1.9 \lesssim z \lesssim 2.9$ (Chapman et al. 2005; Aretxaga et al. 2007), dominating the total star formation rate at this epoch (Pérez-González et al. 2005; Michałowski et al. 2009).

One way to express the results of submm surveys is through number counts of galaxies as a function of flux for each observed wavelength. The shape of these counts has been interpreted as arising from different populations of galaxies whose characteristics evolve over cosmic time (Lagache et al. 2003, 2004; Pearson \& Khan 2009; Le Borgne et al. 2009). These empirical models have successfully reproduced the counts. However, they may be masking a simpler explanation for the departure of the counts from a Schechter distribution at the high-flux end: magnification due to high redshift galaxy clusters and groups (e.g. Blain 1996; Perrotta et al. 2002; Negrello et al. 2007).

Millimeter wavelength surveys have also aimed at detecting galaxy clusters via the Sunyaev Zel'dovich (SZ) effect (Hincks et al. 2008; Carlstrom et al. 2009); the first results, including $\mathrm{CMB}$ power spectra and cluster catalogs, have been released recently (Fowler et al. 2010; Staniszewski et al. 2009; Vanderlinde et al. 2010). The number of detected clusters remains relatively low, pri-

mlima@sas.upenn.edu marily due to the low value of $\sigma_{8}$. However, other effects could be reducing the sensitivity of the surveys (e.g. Lima et al. 2009, 2010).

The South Pole Telescope (SPT) has measured number counts of dusty galaxies at wavelengths $\lambda=1.4 \mathrm{~mm}$ and $2.0 \mathrm{~mm}$ over an area of $87 \mathrm{deg}^{2}$ (Vieira et al. 2009). The observed numbers at the bright end are higher than expected: these galaxies are either at high redshifts and intrinsically exceptionally luminous, or have been magnified by gravitational lensing, or are simply at much lower redshifts than the bulk of the population of submm galaxies. The latter possibility is disfavored by the lack of detected counterparts in other surveys that probed the low redshift population (Vieira et al. 2009). The possibility that these galaxies are at high redshifts and intrinsically bright would require them to be far more luminous than an underlying Schechter-like luminosity function would permit. Thus the favored explanation is that they have typical luminosities for high- $z$ galaxies, but have been magnified by foreground galaxies or clusters. In fact, lensing of high-redshift background submm galaxies has been observed in a number of systems (Smail et al. 1997, 2002; Wilson et al. 2008; Rex et al. 2009; Gonzalez et al. 2009; Swinbank et al. 2010).

In this Letter, we explore the possibility that the existing observed galaxy number counts over a wide range of wavelengths can be reproduced by a single population of galaxies at high-redshift. Foreground galaxy groups and clusters gravitationally lenses the background submm population (Lima et al. 2009) and leads to significant enhancements of the high-flux end to the galaxy counts. In $\S 2$ we describe the lensing magnification formalism, which we then apply to a high- $z$ galaxy population and present results in $\S$. We discuss implications for high- $z$ galaxies and the cluster searches in $\S$ 田

Throughout, we use a fiducial cosmology for a flat universe with parameter values based on the results of the Wilkinson Microwave Anisotropy Probe fifth year data release (WMAP5, Komatsu et al. 2009). The cosmological parameters (and their values) are the normaliza- 


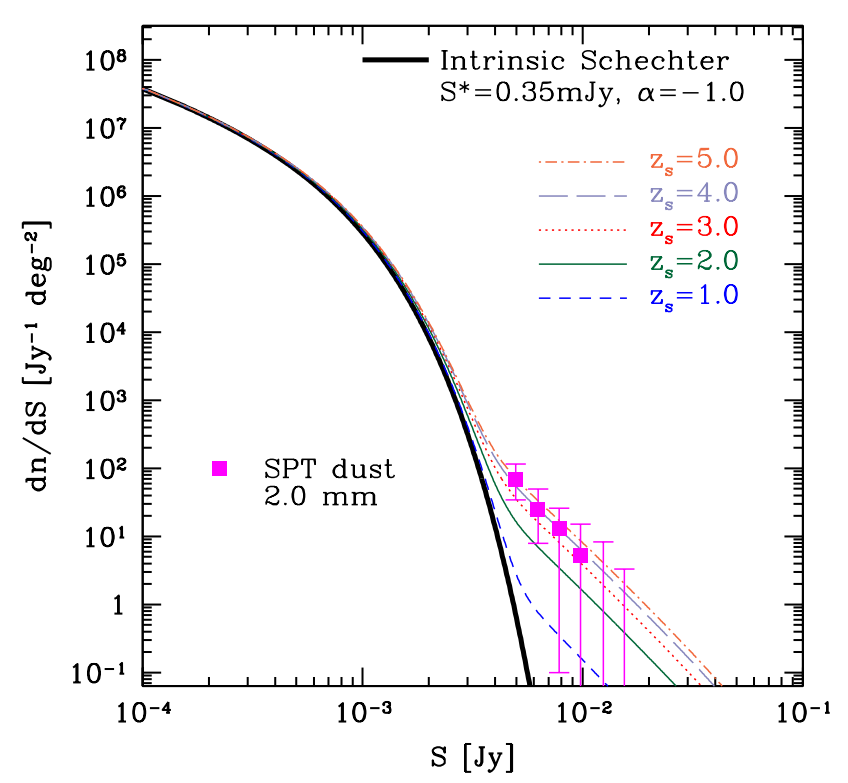

Figure 1. Intrinsic and lensed number counts $d n / d S$ for a Schechter function describing galaxies at different redshifts. Also shown are the observed counts for SPT dusty submm galaxies at $\lambda=2.0 \mathrm{~mm}$, after removal of low-redshift galaxies with IRAS counterparts.

tion of the initial curvature spectrum $\delta_{\zeta}\left(=2.41 \times 10^{-4}\right)$ at $k=0.02 \mathrm{Mpc}^{-1}$ (corresponding to $\sigma_{8}=0.8$ ), its tilt $n(=0.96)$, the baryon density relative to critical $\Omega_{b} h^{2}(=0.023)$, the matter density $\Omega_{\mathrm{m}} h^{2}(=0.13)$, and two dark energy parameters: its density $\Omega_{\mathrm{DE}}(=0.74)$ and equation of state $w(=-1)$, which we assume to be constant. Since lensing effects depend on the abundance of dark matter halos, which is exponentially sensitive to linear matter perturbations, we also consider changes in $\sigma_{8}$ consistent with the WMAP5 errors of $\Delta \sigma_{8} \approx 0.03$. Our central value and uncertainty for $\sigma_{8}$ is consistent with the WMAP7 analysis (Komatsu et al. 2010).

\section{NUMBER COUNTS WITH LENSING MAGNIFICATION}

In recent papers (Lima et al. 2009; Jain \& Lima 2010) we have presented a halo model for calculating the effects of lensing magnification by galaxy groups and clusters. Here we specialize to the case of steep galaxy counts at high redshifts, where lensing effects are quite dramatic. We assume a Schechter function (Schechter 1976) for the intrinsic number density distribution of a population of galaxies

$$
\frac{d n}{d S}=\frac{n^{*}}{S^{*}}\left(\frac{S}{S^{*}}\right)^{\alpha} e^{-S / S^{*}}
$$

where $n^{*}, S^{*}$ and $\alpha$ are free parameters. Due to lensing magnification by intervening halos, the intrinsic $d n / d S$ is changed to its observed counterpart as

$$
\frac{d n_{\mathrm{obs}}\left(S_{\mathrm{obs}}\right)}{d S_{\mathrm{obs}}}=\int d \mu \frac{P(\mu)}{\mu} \frac{d n}{d S}\left(\frac{S_{\mathrm{obs}}}{\mu}\right),
$$

where $\mu$ is the lensing magnification and $P(\mu)$ is its probability for a given galaxy population at redshift $z_{s}$. Conditional probabilities quantify the effects of different magnification ranges on the observed flux density $S_{\text {obs }}$. The integrand of Eq. 2 defines the probability $P\left(\mu \mid S_{\mathrm{obs}}\right)$

$$
P\left(\mu \mid S_{\mathrm{obs}}\right)=\left(\frac{d n_{\mathrm{obs}}\left(S_{\mathrm{obs}}\right)}{d S_{\mathrm{obs}}}\right)^{-1} \frac{P(\mu)}{\mu} \frac{d n}{d S}\left(\frac{S_{\mathrm{obs}}}{\mu}\right),
$$

which can be interpreted as the relative contribution of a given $\mu$ to the total $d n_{\text {obs }} / d S_{\text {obs }}$ at $S_{\text {obs }}$ (Paciga et al. 2008). Similarly $P\left(\mu_{\min } \mid S_{\text {obs }}\right)=\int_{\mu_{\min }}^{\infty} d \mu P\left(\mu \mid S_{\text {obs }}\right)$ measures the integrated contribution from all $\mu>\mu_{\min }$. The mean magnification at a given $S_{\text {obs }}$ is defined as

$$
\langle\mu\rangle\left(S_{\mathrm{obs}}\right)=\int_{0}^{\infty} d \mu \mu P\left(\mu \mid S_{\mathrm{obs}}\right) .
$$

The distribution $P(\mu)$ can be estimated either by raytracing on N-body simulations (e.g. Hilbert et al.|2007), or by semi-analytical methods (e.g. Perrotta et al. 2002; Lima et al. 2009), integrating halo contributions on the line of sight up to the source redshift

$$
P(>\mu)=\int_{0}^{z_{s}} d z_{l} \frac{D_{A}^{2}\left(z_{l}\right)}{H\left(z_{l}\right)} \int_{M_{\mathrm{th}}}^{\infty} d \ln M \frac{d n\left(z_{l}, M\right)}{d \ln M} \Delta \Omega_{\mu},
$$

where $D_{A}$ is the angular diameter distance, $H$ is the Hubble parameter, $d n / d \ln M$ is the halo mass-function and $\Delta \Omega_{\mu}=\Delta \Omega_{\mu}\left(z_{s}, z_{l}, M\right)$ is the cross-section for magnifications larger than $\mu$ produced by halos of mass $M$ at redshift $z_{l}$ on sources at redshift $z_{s}$. The integrand

$$
\frac{d^{2} P(>\mu)}{d \ln M d z_{l}}=\frac{D_{A}^{2}\left(z_{l}\right)}{H\left(z_{l}\right)} \frac{d n\left(z_{l}, M\right)}{d \ln M} \Delta \Omega_{\mu},
$$

gives the range of halo masses and redshifts contributing most to the probability of a specified minimum magnification $\mu$.

In summary, Eqs. 2 and 5 give the total effect on the counts, Eq. 3 indicates which magnifications contribute most to the given $S_{\text {obs }}$ and Eq. 6] tells us which halo masses and redshifts contribute to a given magnification.

We use this halo-model $P(\mu)$ and correct it for a number of effects. First, as described in Lima et al. (2009), we match our $P(\mu)$ at large magnifications to that of raytracing in dark matter simulations (Hilbert et al. 2007) by tuning the ellipticity of our halos. Next, we account for the effect of luminous matter, which can lead to higher densities via gas cooling, using the simulation results of Hilbert et al. (2008). We also correct for the combination of finite source size and multiple image effects. Magnification effects, especially for large magnifications, are sensitive to the value of $\sigma_{8}$ since it affects the abundance of cluster halos. In the next section we discuss how we account for the uncertainties in our model by giving a range for our predictions.

Our analytical calculation of $P(\mu)$ has some advantages over the approach of numerical simulations (we can easily study changes in source redshift, $\sigma_{8}$ and the contribution from different halo masses and redshift), but it also has some limitations. We only use the one-halo term, which is accurate at the high magnifications relevant for the effects considered here but overestimates the lensing contribution at $\mu \sim 1$. We do not include a distribution of ellipticities or halo substructure, which can also increase magnification cross-sections. We have instead 

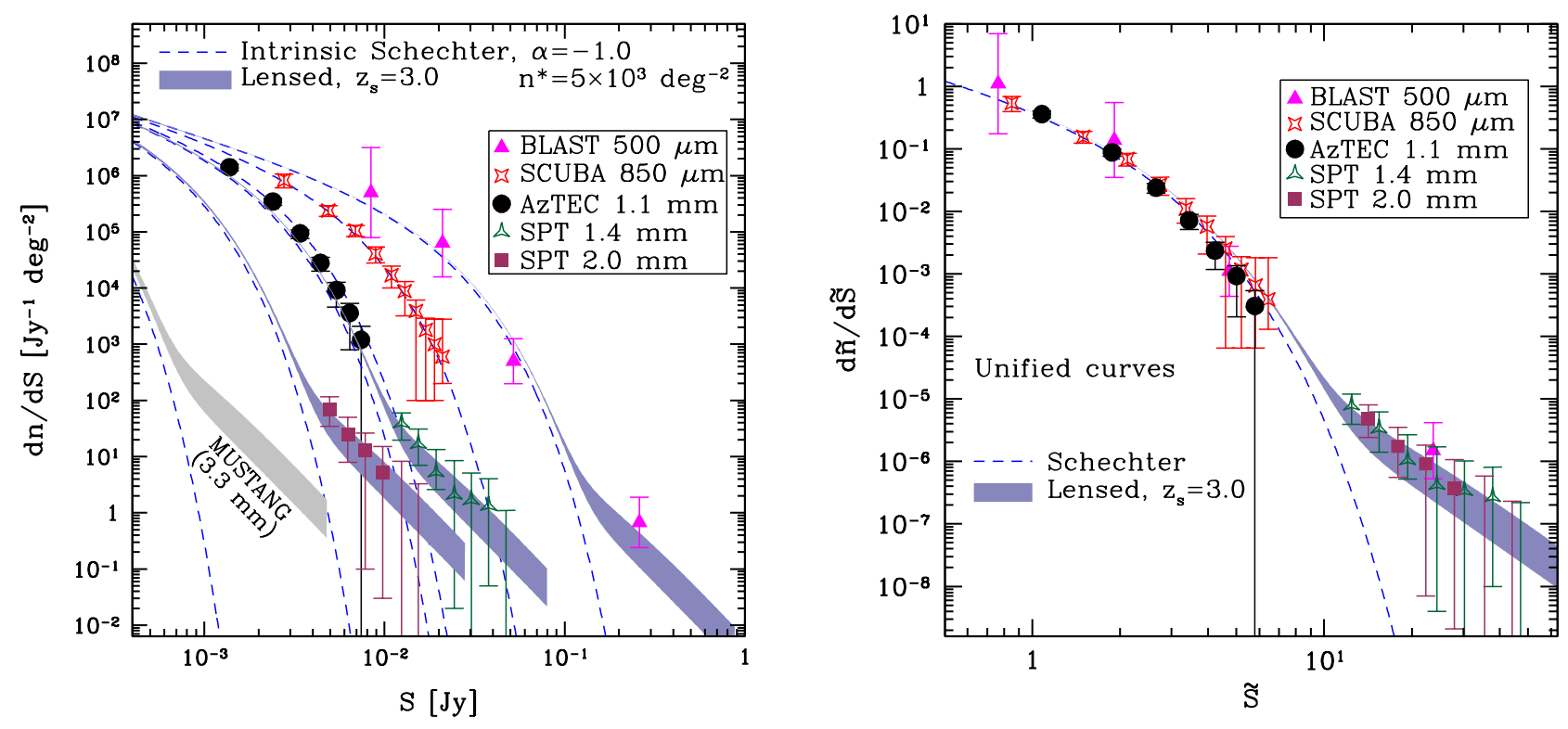

Figure 2. (Left) : Intrinsic and lensed $d n / d S$ for a Schechter function describing galaxies at $z_{s}=3.0$ and different wavelengths. Dashed lines indicate the intrinsic Schechter functions (with different $S^{*}$ values) and the dark shaded regions display the range of lensing predictions, as described in the text. Also shown are observed counts for BLAST at $\lambda=500 \mu \mathrm{m}$, SCUBA at $\lambda=850 \mu \mathrm{m}$, AzTEC at $\lambda=1.1 \mathrm{~mm}$ and SPT at both $\lambda=1.4 \mathrm{~mm}$ and $2.0 \mathrm{~mm}$. The SPT counts are for dusty galaxies, after removal of galaxies with synchrotron emission and galaxies with IRAS counterparts. No similar removal has been applied to the BLAST data, which includes both high and low redshift galaxies. Notice that we do not display the lensing predictions for SCUBA and AzTEC, since these data do not require lensing in the measured fluxes. A prediction for MUSTANG at $\lambda=3.3 \mathrm{~mm}$ is also shown in the light shaded region. (Right) : Unified scaled curves showing $d \tilde{n} / d \tilde{S}$ and the various data points. The only parameter used in the scaling is $S^{*}$; its values at the different wavelengths are shown in Fig. 3

tuned the average halo ellipticity to match the $P(\mu)$ measured in dark matter simulations of Hilbert et al. (2007) (see Lima et al. (2009) for a detailed discussion). And whereas we account for the effects of baryons observed in numerical simulations by Hilbert et al. (2008), these authors note that their simulation still underestimate baryonic effects for halos of smaller masses as they do not predict sufficient numbers of multiply imaged quasars. Finally, the effect of finite source size is very uncertain given the lack of our knowledge about submm galaxies and its sensitivity to the precise caustic structure of the lenses (e.g. Li et al. (2005)). A proper inclusion of all these missing effects would likely increase the magnification probabilities compared to our current model.

\section{RESULTS}

In Fig. 11 we illustrate the lensing effect on an intrinsic Schechter function distribution given by Eq. 1 for sources at different redshifts. In all results presented here, we fix $\alpha=-1.0$ and $n^{*}=5 \times 10^{3} \mathrm{deg}^{-2}$. Changing to $\alpha=-1.5$ does not have a significant effect - while it matches the faint end behavior of $d n / d S$ for the model of Lagache et al. (2004), we preferred to use $\alpha=-1.0$ since this fits better the number counts at shorter wavelengths. With these parameter values, our counts are lower than the model of Lagache et al. (2004) at all $S$, which also ensures that the total flux does not exceed the CIB (Dwek et al. 1998; Fixsen et al. 1998).

Fig. 2 illustrates our main results: we show predicted number counts that include lensing (gray bands), assuming galaxies at $z_{s}=3.0$, along with measured number counts from submm surveys at different wave-

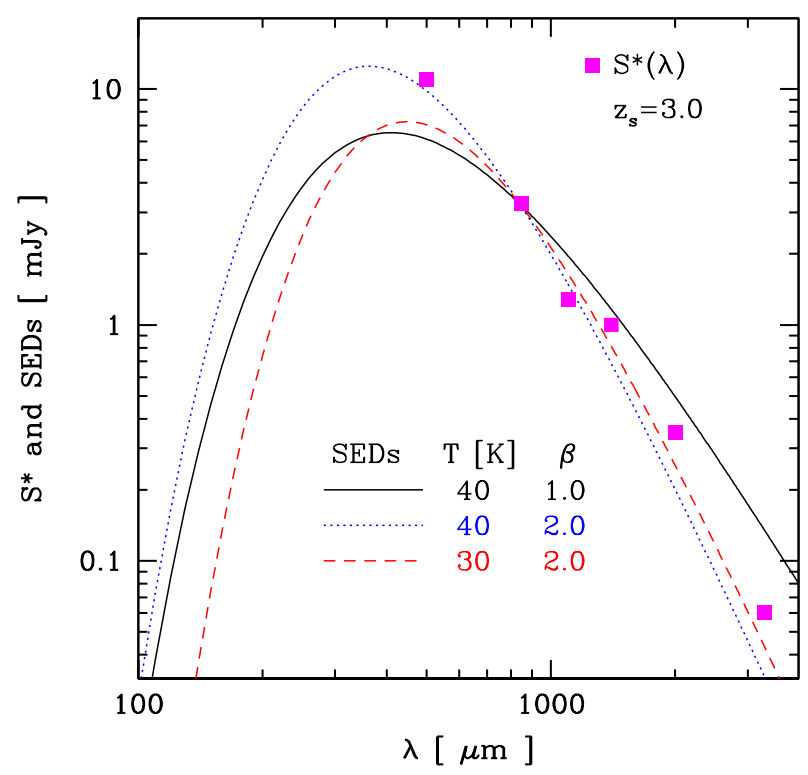

Figure 3. The flux $S^{*}$ in the Schechter function is shown for different surveys at various wavelengths (symbols). The three curves show the expected scaling for submm galaxy SEDs at $z_{s}=3.0$ for different values of spectral index $\beta$ and temperature $T$. The curves are normalized at the value of $S^{*}$ for $\lambda=850 \mu \mathrm{m}$.

lengths. As indicated in the panels, these are: BLAST at $\lambda=500 \mu \mathrm{m}$ (Devlin et al. 2009), SCUBA at $\lambda=850 \mu \mathrm{m}$ (Coppin et al. 2006), AzTEC at $1.1 \mathrm{~mm}$ 

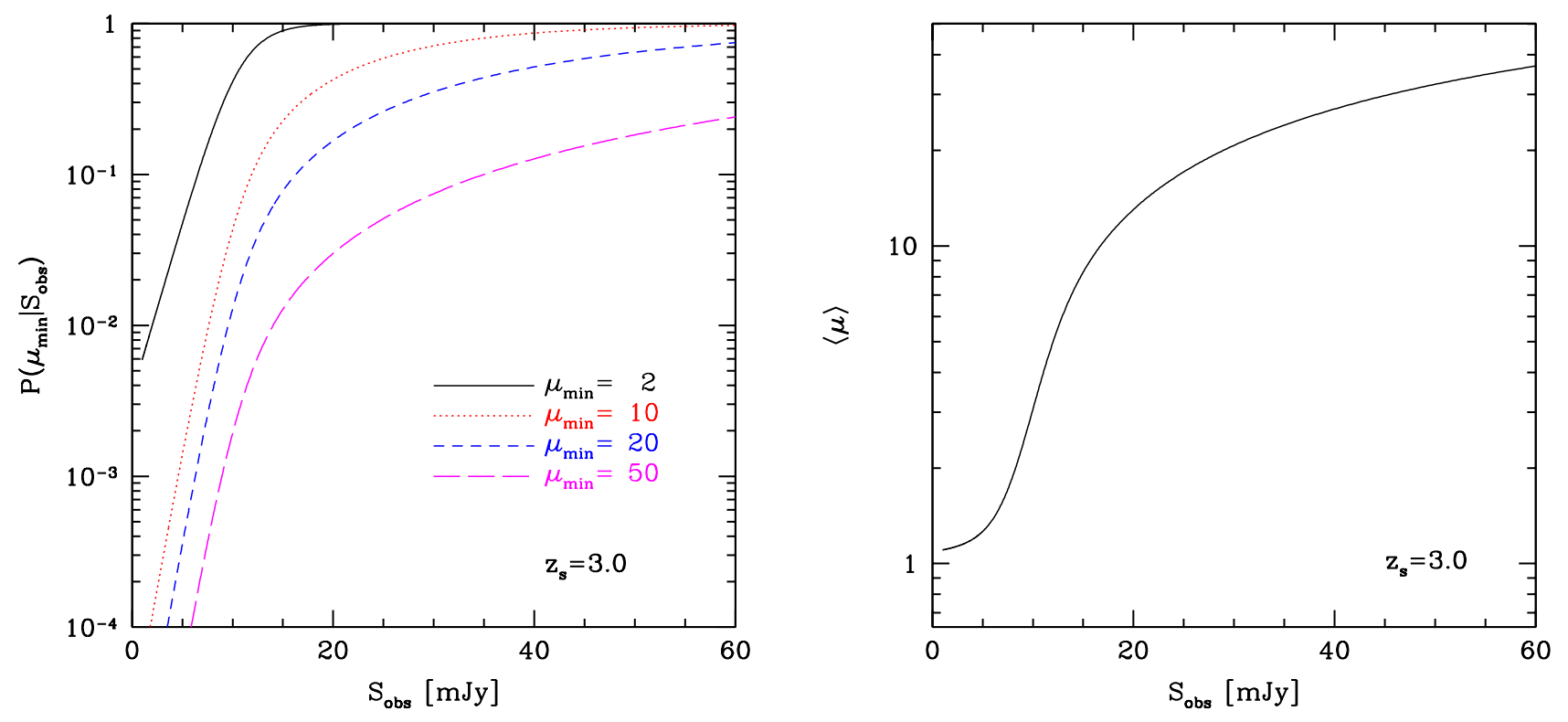

Figure 4. Probability $P\left(\mu_{\min } \mid S_{\text {obs }}\right)$ of the minimum magnification $\mu_{\min }$, given an observed flux density $S_{\text {obs }}$ (left panel), and the corresponding average magnification $\langle\mu\rangle$ (right panel). We assume a Schechter function describing galaxies at $z_{s}=3.0$ which, after lensing, predicts counts consistent with those of SPT dusty submm galaxies at $\lambda=1.4 \mathrm{~mm}$ (see Fig. 2.

(Austermann et al. 2010) and SPT dusty submm galaxies at $\lambda=1.4 \mathrm{~mm}$ and $2.0 \mathrm{~mm}$ (Vieira et al. 2009). In all our results, the SPT number counts correspond to those of Vieira et al. (2009), after removing both synchrotron emission galaxies as well as low-redshift galaxies that have matches with galaxies in the Infrared Astronomy Satellite survey (IRAS, Moshir et al. 1992; Fisher et al. 1995; Oliver et al. 1996). Predictions are shown by bands rather than curves to reflect the uncertainties in the model as discussed below. The intrinsic luminosity function is the Schechter function described above. All the data sets can be fit by changing the single parameter $S^{*}$ once lensing magnification is included. This remarkable result implies that, within the measurement and theoretical uncertainties, a single high- $z$ population of galaxies is sufficient to describe all the observations. The high flux measurements of BLAST and SPT are fit by highly magnified galaxies - if these counts were dominated by a population of a different galaxy type, it would be a coincidence that their relative counts fit the same scaling with wavelength as the fainter ("normal") population. Finally, we also show the prediction for the Multiplexed Squid TES Array at $90 \mathrm{GHz}(3.3 \mathrm{~mm})$ (MUSTANG, Mason et al. 2006) which has begun operating on the Green Bank Telescope.

The lower bound for the predictions in Fig. 2 uses $\sigma_{8}=0.77$ while the upper bound uses $\sigma_{8}=0.83-$ these reflect the uncertainties in the WMAP results as discussed above. The enhancement due to baryons is factored into these predictions following Hilbert et al. (2008), though it is likely to be an underestimate of baryonic effects as discussed above in Section 2. The effect of source sizes and multiple imaging is uncertain; we simply assume that due to the finite source size the effective magnification is reduced by $50 \%$ to $25 \%$ (for the lower and upper bounds respectively)
The right panel of Fig. 2 shows all points rescaled by plotting

$$
\frac{d \tilde{n}}{d \tilde{S}}=\tilde{S}^{\alpha} e^{-\tilde{S}},
$$

where $\tilde{n}=n / n^{*}$ and $\tilde{S}=S / S^{*}$. In Fig. 3 we show values of $S^{*}$ used for each wavelength. We compared the frequency scaling of $S^{*}$ with that of a typical Spectral Energy Distribution (SED) of submm galaxies $\operatorname{SED}(\lambda) \propto$ $\epsilon(\lambda) B(T, \lambda)$, redshifted to $z_{s}=3$ with emissivity $\epsilon(\lambda)=$ $1-\exp \left[\left(-\lambda_{0} / \lambda\right)^{\beta}\right]$ and blackbody spectrum $B(T, \lambda)$ at temperature $T$. The values of $S^{*}$ are consistent with $\beta=1-2$ and $T=30-40 K$, as expected for high redshift submm galaxies.

The fit to the BLAST counts at $\lambda=500,350$ and $250 \mu \mathrm{m}$ falls further below the high flux measurement at shorter wavelengths, suggesting the need for a lower redshift population. Indeed, Eales et al. (2009) have identified the radio and $24 \mu \mathrm{m}$ counterparts of the bright BLAST sources. At $250 \mu \mathrm{m}$ almost all of the bright sources are identified as being at redshifts lower than 1 . One third to one half of the sources in the highest flux bin at $500 \mu \mathrm{m}$ come from sources with $z<1$. Removing these would lower the corresponding point in Fig. 2 in agreement with the model. The remaining sources at $500 \mu \mathrm{m}$ (and less than a tenth of the sources at $250 \mu \mathrm{m}$ ) are likely the result of lensing. Similar results should be expected with the upcoming release of the large-area Herschel surveys.

Since the lensed distributions at $z_{s}=3.0$ are consistent with SPT data points at both wavelengths, we study the range of magnifications and halo masses that would be contributing most in this case. As we consider larger values of $S_{\text {obs }}$, in particular $S_{\text {obs }} / S^{*} \gtrsim 10$, the observed sources come from intrinsically low flux sources which have been magnified significantly. Fig. 4 shows 


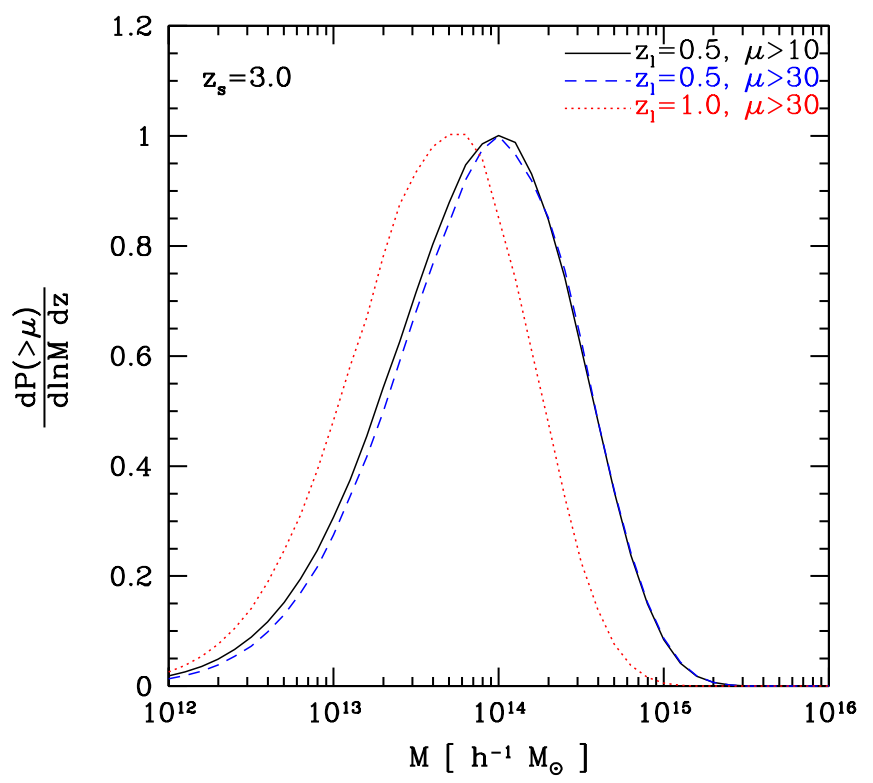

Figure 5. The integrand $d^{2} P(>\mu) / d \ln M d z_{l}$ as a function of halo mass $M$ for different values of $\mu$ and $z_{l}$. We have arbitrarily normalized these curves for better visualization. Note that the peak lensing contribution for source galaxies at $z_{s}=3$ comes from lens halos at $z_{l} \sim 0.5$. This does not include baryonic effects, which boost the contribution from $M \lesssim 10^{13} h^{-1} M_{\odot}$ halos.

$P\left(\mu_{\min } \mid S_{\text {obs }}\right)$ and $\langle\mu\rangle$ as a function of $S_{\text {obs }}$ for $\operatorname{SPT}(\lambda=$ $1.4 \mathrm{~mm})$ and indicates magnifications that contribute most at each $S_{\text {obs }}$. For instance, for $S_{\text {obs }}=20-40 \mathrm{mJy}$, the right panel shows that $\langle\mu\rangle \sim 20-30$. Another way to see this is via $P\left(\mu_{\min } \mid S_{\text {obs }}\right)$ (left panel), which integrates out the effects above a certain $\mu_{\text {min }}$, and shows that for $S_{\text {obs }}=20-40 \mathrm{mJy}$, we have $P\left(\mu_{\min } \mid S_{\text {obs }}\right)>0.5$ for $\mu_{\min } \sim 10-20$.

These results imply that magnifications of $10-30$ are necessary to explain the boost in $d n / d S$ at $S_{\text {obs }} \sim$ $10-40 \mathrm{mJy}$, if it is due to lensing of an intrinsic Schechter distribution. Note that due to the finite size of submm galaxies, their magnifications must have a cut-off, which has been estimated to be in the range $\mu \sim 10-40$ (Perrotta et al. 2002) for galaxy lenses, and is probably a factor of two or so larger for more massive lenses. Indeed galaxies have been measured with estimated magnifications of at least $\sim 45$ (Paciga et al. 2008; Kneib et al. 2004).

In Fig. 5 we show $d^{2} P / d \ln M d z_{l}$ as a function of halo mass for different values of $\mu_{\min }$ and $z_{l}$. This indicates that halo masses above $10^{13} h^{-1} M_{\odot}$ contribute significantly to magnifications of $10-30$, with most of the contribution coming from $\sim 10^{14} h^{-1} M_{\odot}$. Note that this does not include baryonic effects, which boost the contribution from lower mass $\left(\lesssim 10^{13} h^{-1} M_{\odot}\right)$ halos as discussed in Section 2.

We have assumed that all source galaxies are at a fixed redshift $z_{s}$, whereas in reality they have a redshift distribution which needs to be incorporated in the computation. The curves at different source redshifts shown in Fig. 1 provide approximate limits for what we can expect from a redshift distribution of source galaxies. Our results imply that the bulk of the population of submil- limeter galaxies are at redshifts $z \gtrsim 2$. The alternative explanation for the high flux measurements is to have a significant fraction of galaxies at very low $z$, which would easily have been observed in surveys such as IRAS (indeed the SPT data shown remove the small fraction of such sources). And as shown in Fig. 11 galaxies at higher redshift but still at $z \lesssim 1$ would not match the measurements as the magnification boost is insufficient (again within the context of an underlying Schechter distribution).

\section{DISCUSSION}

We have considered the possibility that bright millimeter and submillimeter galaxy counts arise largely from a galaxy population at high redshifts that is lensed by intervening galaxy groups and clusters. Our model predictions match the counts from $500 \mu \mathrm{m}$ to $2 \mathrm{~mm}$ from the BLAST, SCUBA, AzTEC and SPT surveys (see Fig. 2). We find that the high flux SPT number counts can be explained by highly magnified galaxies from this high$z$ population (once the known, low- $z$ counterparts detected in IRAS are removed). This high- $z$ galaxy population is described by a Schechter luminosity function with $L^{*} \sim 2.5 \times 10^{12} L_{\odot}$ and a source redshift $z_{s}=3.0$. Our model predictions fit the data for $500 \mu \mathrm{m}<\lambda<2 \mathrm{~mm}$ by varying $S^{*}$ with wavelength within the range of typical submillimeter galaxy SEDs (see Fig. 3).

Our model has some simplifying assumptions, such as the fixed source redshift $z_{s}=3$, and there are significant measurement and theoretical uncertainties. A complete analysis would require a more detailed treatment of the lensing and inclusion of the measured galaxy clustering. In addition, it has been established that at the shorter wavelengths probed by BLAST, an increasing fraction of sources lie at low- $z$ - hence we can expect some smooth variation in the fraction of low- $z$ galaxies with observed wavelength.

Nevertheless our results in Fig. 2 imply that current number counts do not require an additional galaxy population to explain the high flux measurements. Such a population has been invoked in theoretical models (e.g. Lagache et al. 2003) and suggested as a possible explanation (as well as lensing of the high- $z$ population) for the recent SPT measurements (Vieira et al. 2009). Indeed, adding a significant fraction of a second population could cause the predictions to exceed the measured counts once magnification effects for the high- $z$ population are included. It would also be difficult to explain how the scaling with wavelength of the high flux number counts is the same as the lower flux counts if they came from different galaxy populations. Since lensing does not depend on frequency, our model naturally follows this common scaling.

Current SZ surveys with sensitivities of $3-7 \times$ $10^{14} h^{-1} M_{\odot}$ cannot detect most halos that produce this lensing contribution. Conversely, looking for extremely bright objects in millimeter and submillimeter wavelengths provides a way to find high- $z$ lensing halos associated with galaxy groups and clusters. Note that the number of halos that can be found in this way is only a small fraction of all halos within a given mass range. As discussed above, our model most likely underestimates the contribution from halos with $M \lesssim 10^{13} M_{\odot}$. It is therefore of great interest to investigate the lenses corre- 
sponding to the bright sources in current data.

Follow up observations with optical telescopes should be able to identify lensing group/cluster candidates up to $z \simeq 1$. These clusters and groups host Brightest Cluster Galaxies with luminosities $L \sim 10^{11}-10^{12} L_{\odot}$, based on the low- $z$ results of Johnston et al. (2007) from the Sloan Digital Sky Survey. Multi-band optical imaging with limiting magnitude of $23-24$ (for the $r$ band) would enable identification of these groups and clusters. Conversely, targeted observations of the high magnification regions of known strong lensing clusters could provide detections of faint submm galaxies (which would lie below the detection threshold without the magnification boost). Similar to the use of clusters as gravitational telescopes in optical imaging, this may also help resolve submm galaxies. Planned observations with AzTEC and the Large Millimeter Telescope have considered such an approach (David Hughes, private communication).

We thank David Hughes, Roxana Lupu, Joaquin Vieira, Kim Scott, Ian Smail, Eric Switzer and various members of the ACT collaboration for useful discussions. We benefited from discussions on strong lensing effects with Matthias Bartelmann, Gary Bernstein, Neal Dalal and Ravi Sheth. We are very grateful to Stefan Hilbert for sharing his simulation results and Eric Switzer for providing the SPT data. This work was supported in part by an NSF-PIRE grant and AST-0607667.

\section{REFERENCES}

Aretxaga, I., et al. 2007, MNRAS, 379, 1571

Austermann, J. E., et al. 2010, MNRAS, 401, 160

Barger, A. J., Cowie, L. L., Sanders, D. B., Fulton, E., Taniguchi, Y., Sato, Y., Kawara, K., \& Okuda, H. 1998, Nature, 394, 248

Blain, A. W. 1996, MNRAS, 283, 1340

Carlstrom, J. E., et al. 2009, ArXiv e-prints

Chapman, S. C., Blain, A. W., Smail, I., \& Ivison, R. J. 2005, ApJ, 622, 772

Coppin, K., et al. 2006, MNRAS, 372, 1621

Devlin, M. J., et al. 2009, Nature, 458, 737

Dwek, E., et al. 1998, ApJ, 508, 106

Eales, S., et al. 2009, ApJ, 707, 1779

Fisher, K. B., Huchra, J. P., Strauss, M. A., Davis, M., Yahil, A., \& Schlegel, D. 1995, ApJS, 100, 69

Fixsen, D. J., Cheng, E. S., Gales, J. M., Mather, J. C., Shafer, R. A., \& Wright, E. L. 1996, ApJ, 473, 576

Fixsen, D. J., Dwek, E., Mather, J. C., Bennett, C. L., \& Shafer, R. A. 1998, ApJ, 508, 123

Fowler, J. W., et al. 2010, ArXiv e-prints
Gonzalez, A. H., Clowe, D., Bradač, M., Zaritsky, D., Jones, C., \& Markevitch, M. 2009, ApJ, 691, 525

Greve, T. R., Ivison, R. J., Bertoldi, F., Stevens, J. A., Dunlop, J. S., Lutz, D., \& Carilli, C. L. 2004, MNRAS, 354, 779

Hilbert, S., White, S. D. M., Hartlap, J., \& Schneider, P. 2007, MNRAS, 382, 121

-. 2008, MNRAS, 386, 1845

Hincks, A. D., et al. 2008, in Society of Photo-Optical Instrumentation Engineers (SPIE) Conference Series, Vol. 7020, Society of Photo-Optical Instrumentation Engineers (SPIE) Conference Series

Hughes, D. H., et al. 1998, Nature, 394, 241

Jain, B., \& Lima, M. 2010, ArXiv e-prints

Johnston, D. E., et al. 2007, ArXiv e-prints

Kneib, J., van der Werf, P. P., Kraiberg Knudsen, K., Smail, I., Blain, A., Frayer, D., Barnard, V., \& Ivison, R. 2004, MNRAS, 349,1211

Komatsu, E., et al. 2009, ApJS, 180, 330

-. 2010, ArXiv e-prints

Lagache, G., Dole, H., \& Puget, J. 2003, MNRAS, 338, 555

Lagache, G., et al. 2004, ApJS, 154, 112

Le Borgne, D., Elbaz, D., Ocvirk, P., \& Pichon, C. 2009, A\&A, 504,727

Li, G., Mao, S., Jing, Y. P., Bartelmann, M., Kang, X., \& Meneghetti, M. 2005, ApJ, 635, 795

Lima, M., Jain, B., \& Devlin, M. 2009, ArXiv e-prints

Lima, M., et al. 2010, in prep.

Mason, B. S., Dicker, S., Korngut, P., Benford, D., Devlin, M., Irwin, K., Moseley, H., \& MUSTANG collaboration. 2006, in Bulletin of the American Astronomical Society, Vol. 38, 1015-+

Michałowski, M. J., Hjorth, J., \& Watson, D. 2009, ArXiv e-prints

Moshir, M., Kopman, G., \& Conrow, T. A. O. 1992, IRAS Faint Source Survey, Explanatory supplement version 2, ed. Moshir, M., Kopman, G., \& Conrow, T. A. O.

Negrello, M., Perrotta, F., González-Nuevo, J., Silva, L., de Zotti, G., Granato, G. L., Baccigalupi, C., \& Danese, L. 2007, MNRAS, 377, 1557

Oliver, S. J., et al. 1996, MNRAS, 280, 673

Paciga, G., Scott, D., \& Chapin, E. L. 2008, ArXiv e-prints

Pearson, C., \& Khan, S. A. 2009, MNRAS, 399, L11

Pérez-González, P. G., et al. 2005, ApJ, 630, 82

Perrotta, F., Baccigalupi, C., Bartelmann, M., De Zotti, G., \& Granato, G. L. 2002, MNRAS, 329, 445

Pope, A., et al. 2006, MNRAS, 370, 1185

Rex, M., et al. 2009, ApJ, 703, 348

Schechter, P. 1976, ApJ, 203, 297

Smail, I., Ivison, R. J., \& Blain, A. W. 1997, ApJ, 490, L5+

Smail, I., Ivison, R. J., Blain, A. W., \& Kneib, J. 2002, MNRAS, 331,495

Staniszewski, Z., et al. 2009, ApJ, 701, 32

Swinbank, M., et al. 2010, ArXiv e-prints

Vanderlinde, K., et al. 2010, ArXiv e-prints

Vieira, J. D., et al. 2009, ArXiv e-prints

Wilson, G. W., et al. 2008, MNRAS, 390, 1061 